\title{
Open Quotient Influence on Performances of Pathological Voice Classification by SVM
}

\author{
Asma Belhaj ${ }^{*}$, Aïcha Bouzid* ${ }^{*}$ Noureddine Ellouze*
}

\begin{abstract}
The aim of this paper is to study the efeect of the open quotient in the classification of pathological voices. The Open quotient is a glottal voicing parameter representing the ratio of the open phase by the pitch period. This parameter is computed knowing the GCI and the GOI detected by the MPM. The classification is operated on MEII pathological database using SVM classifiers multi-class one-against-all. We consider a binary classification into normal and pathological voices for female speakers and for all speakers of the database, and a threeclass classification into edema, nodule and normal voices for the female speakers.
\end{abstract}

Keywords - Pathological Voices, SVM, Open Quotient.

\section{I.Introduction}

Voice Pathologies affects speaking capabilities and have a large impact on both professional and social life. In recent years, researchers in laryngology and speech science have become increasingly interested in the acoustic characteristics of normal and pathological voices [1-3]. Nowadays, dysphonia is a disease affecting more and more subjects due to the disturbance of the produced speech, while the larynx is involved in the phonation. We can distinguish various types of dysphonia :organic, dysfunctional and neurological dysphonias.

The assessment of pathological voices can be very relevant for both diagnosis and therapy evaluation. The assessment of the voice quality can be made by a diagnostician or by a direct examination using the laryngostroboscopy. The diagnosis may be performed following two different approaches: the perceptive and the objective ones. The perceptive assessment consists of qualifying the voice pathologies by listening to the patient. The evaluation is performed by rating the speech samples on GRBAS grade scale [4]. The objective analysis consists of quantifying the voice pathologies by acoustical, aerodynamic, and physiological measurements. It offers also the advantages to be, cheaper, faster, and more comfortable for the patient than methods like the electro-glottography (EGG) [5] stroboscopy imaging [6] or high-speed camera [7].

Given the complex and subjective nature of the personal listening, researchers have developed various tools for establishing a diagnosis. Different acoustic evaluation methods of pathological voices have been proposed. Among them, the automatic classification of pathological voice has received a considerable attention. The most important classifiers used in the classification of pathological voices are: neural networks (RN), the Gaussian mixture model (GMM), the hidden Markov model (HMM), and the support vector machines (SVM). Many studies have proposed a binary classification into normal/pathological[8,10]. Besides the SVM classifier using specific parameters, have achieved the best performance. However, the classification between the pathologies is operated in few works [9] and the results are not sufficiently efficient.

In this work, we consider two types of classification using the classic features MFCC, $\Delta, \Delta \Delta$, the energy, the fundamental frequ

ency added to the open quotient. The open quotient is defined as the ratio of the open phase by the pitch period. The open phase is the time interval separating the glottal opening instant (GOI) and the following glottal closure instant (GCI).

This paper is organized as follows. In Section 2, we give a brief description of the classic features used in the pathologic voice classification. Section 3 presents the most important classifiers. In section 4, we describe the MEII database used in this work. Section 5 presents the features extracted from the speech signal and the open quotient. In Section 6, we present the GCI and GCI detection, section 7 present the open quotient estimation. In section 8 we give the binary and the multi-class SVM classification. The results are presented in section 9. Performances of classification are given in section 10. Finally conclusion and future work are drawn in section 11.

\section{Classic Features in Pathologic Voice Discrimination}

The classic features used in the classification of pathologic voices are inspired from the cues used in the field of the speech recognition that are essentially the fundamental frequency $\mathrm{F} 0$, the mel frequency cepstral coefficients (MFCC), first and second derivatives, energy, harmonic to noise ratio (HNR).

\section{Asma Belhaj}

Laboratory of Signal, Images and Information Technology, Tunis El Manar University, National Engineering School of Tunis, TUNISIA

Aïcha Bouzid

Laboratory of Signal, Images and Information Technology, Tunis El Manar University, National Engineering School of Tunis, TUNISIA

Noureddine Ellouze

Laboratory of Signal, Images and Information Technology, Tunis El Manar University, National Engineering School of Tunis, TUNISIA 
The subject of this section is the overview of the most common features involved in the pathological voice assessment.

\section{A. Mel-Frequency Cepstral Coefficients MFCC}

MFCCs are one of the most widely-used features to reduce the redundancy of the speech signal to be used in domains like recognition or coding [11]. These coefficients are computed by weighting the Fourier Transform of the signal by a MEL filterbank, then computing the cepstrum from this weighted spectrum and finally the Discrete Cosine Transform (DCT) of this cepstrum.

\section{B. Fundamental Frequency FO}

It's an obvious parameter describing the speech voicing state. This parameter is used in most of the studies, sometimes in conjunction with the Mel-Frequency Cepstral Coefficients (MFCC).

\section{Harmonic to Noise Ratio HNR}

This parameter is defined as the log ratio of the energy of the periodic and aperiodic components [12]. It can be computed with different approaches. In fact, some methods are based on a model in which speech is assumed to be composed of a periodic component and an aperiodic component [13, 14] while other use the short-time autocorrelation function [15]. All these approaches are based on the estimation of the fundamental frequency. It is used in [16] for the discrimination between normal and pathological voices using the MEEI database. The same measure is used in [17] to show that HMM is able to classify different voice qualities and in $[18,19]$ to discriminate normal and pathological voices through a telephone channel.

\section{Acoustic Features from MDVP Software}

The Multi-Dimensional Voice Program (MDVP) is software produced by KayPentax Corp [20]. This software provides some acoustic descriptors defined in [21] and stored with speech samples in the MEEI database. The parameters concern the perturbation of the fundamental frequency and the amplitude of the signal. Some proposed classification systems use these acoustic descriptors computed directly from the MDVP software [22, 23]. Some other systems use features inspired from those computed by MDVP software [18, 19], meaning that their definitions is taken or inspired from [20].

\section{Classifiers used in Normal/Pathological Voices Discrimination}

The aim of this section is to describe the different types of classifiers used in the voice pathology assessment. Their structure and behavior are briefly presented.

\section{A. Gaussian Mixture Model}

The Gaussian Mixture Modeling (GMM) is widely used in Automatic Speaker Recognition, where it acts as a supervised classification system. It is adapted from speaker identification to a classification in one grade of GRBAS scale (from 0 (normal) to 3). The GMM classification system operates following three steps [24].

(i) Parametrization, (ii) Model training and (iii) Classification: when a speech sample has to be classified, the likelihood between this sample and each GMM is estimated and the decision relies on the maximum between these likelihoods.

For the normal/pathological classification, $95 \%$ of normal subjects and $81.7 \%$ of pathological ones are correctly classified. For the grade classification, $95 \%$ is obtained for the grade 0 corresponding to the normal subjects while a loss of performance is observed for the pathological ones, specially between adjacent grades. The same system is used in [25] to determine which kind of information is better suited to the classification of the four grades.

\section{B. Support Vector Machines}

Support Vector Machines (SVMs) [26] are a well-known classifier used in problems of classification, regression, and novelty detection. Recent researches use this classifier in discrimination between normal and pathological samples. For example, [2] proposes to use a set of features consisting of 11 MFCC coefficients, HNR (Harmonic to Noise Ratio), NNE (Normalized Noise Energy), GNE (Glottal to Noise Excitation), Energy, and their first derivatives.

The classifier is trained on the vowels /a/ from the pathological corpus of MEII Database (53 normal samples and 77 pathological samples) and the average correct classification rate is $95.12 \%$. The SVM classifier using features extracted from wavelet transform of speech samples to discriminate between normal and pathological voices [27]. The correct classification rate is this time $97.5 \%$ for normal voices and $100 \%$ for pathological ones.

\section{Neural Networks}

The Artificial Neural Networks are ones of the widely used classifier in various domains, as pattern classification and recognition and particularly speech recognition. Basically this type of classifier can be viewed as an interconnexion between simple small units, the neurons, designed to model to some extent the behaviour of human brain. In [28], this type of classifier is applied on MEII database to distinguish between normal and pathological samples. The input layer is composed of 26 neurons corresponding to 26 acoustic descriptors given by the MDVP software. Besides, the classifier is composed of 1-hidden layer and 1-neuron output layer for normal or pathologic decision. The average correct classification rate is 94\% when HNR, VTI, and ShdB are used as input features. The discrimination between normal and pathological samples is also operated on a database of 5 spanish sustained vowels (100 normal samples and 68 pathological samples) [29]. Each vowel is treated by a neural network which takes as input 
classic parameters and others extracted from the bi-coherence. The decisions from the 5 networks are then combined to decide if the input sample is healthy or not. The correct classification rate is $94.4 \%$ for the classic parameters and is increased of $4 \%$ when the others ones are added.

\section{MEII Database}

Kay Elemetrics Voice Disorder Database was developed by the Massachusetts Eye and Ear Infirmary (MEEI) Voice and Speech Labs (Kay Elemetrics Corp., 1994). The acoustic samples are sustained phonations of vowel [a] (3 - 4 s long) and the first 12 seconds of the Rainbow Passage spoken by normophonic subjects and patients with organic, neurological, traumatic, and psychogenic voice disorders at different stages (from early to fully developed). The speech samples have been recorded in a controlled environment at $25 \mathrm{kHz}$ or $50 \mathrm{kHz}$ and 16 bits of resolution. We have considered a subset comprising 53 normal and 169 pathological voices omitting recordings devoid of a diagnosis and balancing samples with regard to sex and chronological age [30] as shown in table 1.

TABLE1. Details of the recordings used in this study

\begin{tabular}{|c|c|c|c|c|c|c|c|c|}
\hline & \multicolumn{2}{|c|}{ Subjets } & \multicolumn{2}{|c|}{ Range(yaers) } & \multicolumn{2}{|c|}{ Average (years) } & \multicolumn{2}{|c|}{$\begin{array}{l}\text { Standard } \\
\text { deviation (years) }\end{array}$} \\
\hline & Male & Female & Male & Female & Male & Female & Male & Female \\
\hline \multicolumn{9}{|l|}{ MEII } \\
\hline Normal & 21 & 32 & $26 / 58$ & $22 / 52$ & 38.8 & 34.2 & 8.49 & 7.87 \\
\hline $\begin{array}{l}\text { Patho- } \\
\text { logic }\end{array}$ & 70 & 103 & $26 / 58$ & $21 / 51$ & 41.7 & 37.6 & 9.38 & 8.19 \\
\hline
\end{tabular}

\section{v. Feature Extraction}

The feature extraction constitutes the first step in a classification system.

This step consists in determining the MFCCC coefficients, the derivations $\Delta, \Delta \Delta$, the energy, the fundamental frequency F0 and the open quotient Oq. The first coefficients are computed using the melcepst function provided by the voicebox toolbox [32]. The speech signals are divided into frames of $46.44 \mathrm{~ms}$ and with a half recovery. The sampling frequency of MEII database is $25 \mathrm{kHz}$, so the window contains 1161 samples with an overlap of 581 samples, or a sampling frequency of $50 \mathrm{kHz}$, the window contains 2322 samples with an overlap of 1161 samples. The open quotient and the fundamental frequency are determined by the glottal closure instant (GCI) and the glottal opening instant (GOI) detected by the multi-scale product (MP) of the speech signal.

\section{vi. Multiscale Product for GCI and GOI detection}

The algorithm shall calculate the product of the coefficients of the wavelet transform for different successive scales as shown in figure 2:

$$
p(n)=\prod_{j=1}^{3} \psi_{2^{j}}(f(n))
$$

Where $\psi_{2^{j}}(f(n))$ is the wavelet transform of function $f(n)$ at scale $2^{j}$.

We note in the cross scale product two types of peaks, minima corresponding to GCI are the most distinguishable, and maxima related to GOI are considerably weaker but discernible. The GOI is the maximum detected between two GCI, as shown in fig 1 and 2 .

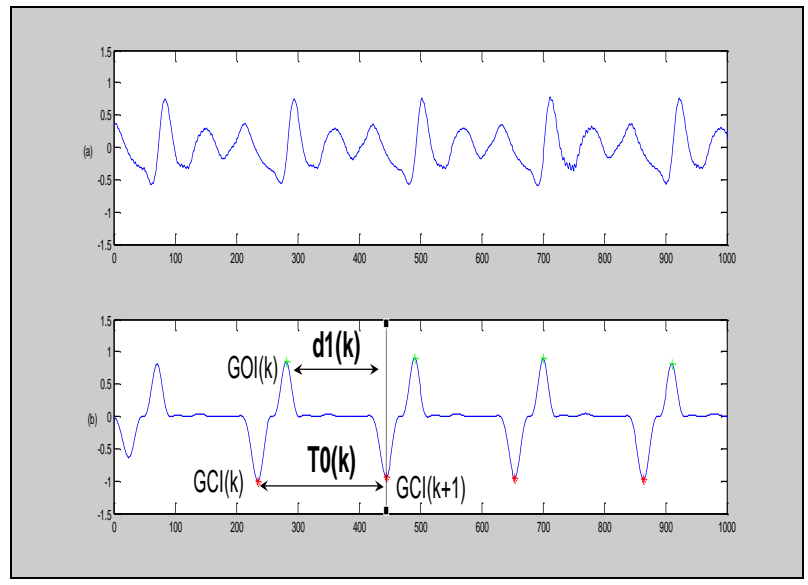

Figure1. Speech normal voice corresponding to a sustained vowel /a/ extracted from AXH1 pronounced by a female speaker and its MP of MEEI database.

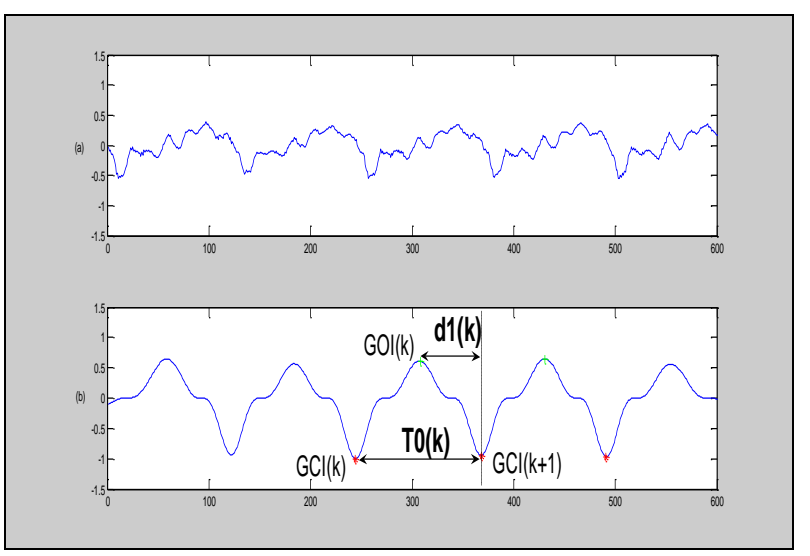

Figure2. Speech pathological voice corresponding to a sustained vowel /a/ extracted from paralysis AXT13 pronounced by a male speaker and its MP of MEEI database.

\section{vII. Open quotient Oq and PITCH estimation $\mathbf{T}_{0}$}

For our databases, we use two combinations of scales $\mathrm{s} 1=$ $2, \mathrm{~s} 2=5 / 2, \mathrm{~s} 3=3$ for women and a second $\mathrm{s} 1^{\prime}=3, \mathrm{~s} 2{ }^{\prime}=4$,

s3 '= 5 for men. This difference in the choice of scales is that the discontinuities at the GCI and GOI had more in men 
than in women as shown in figure 3 and 4. The wavelet used in this work is the quadratic spline function. For each analysis window, we locate the GCIs and GOIs, then we calculate the instantaneous values of the fundamental frequency and the open quotient from the following equations.

The local pitch period is given by the following formula:

$$
T_{0}(k)=G C I(k+1)-G C I(k)
$$

The local fundamental frequency F0 $(\mathrm{k})$ is given by the inverse of the pitch period:

$$
F_{0}(k)=\frac{1}{T_{0}(k)}
$$

The open quotient is defined as the ratio of the duration of the open phase by the fundamental period.

$$
O_{q}(k)=\frac{G C I(k+1)-G O I(k)}{T_{0}(k)}
$$

The mean values in the window are calculated according to the following relationships:

$$
\begin{aligned}
& F_{\mathrm{O}}^{i}=\frac{1}{N} \sum_{k=1}^{N} F_{\mathrm{O}}^{i}(k) \\
& O_{q_{0}}^{i}=\frac{1}{N} \sum_{k=1}^{N} O_{q}^{i}(k) \\
& J i F_{\mathrm{O}}^{i}=\frac{1}{N-1} \sum_{k=1}^{N-1} F_{\mathrm{O}}^{i}(k+1)-F_{\mathrm{O}}^{i}(k) \\
& J_{i} O_{q_{\mathrm{O}}}{ }^{i}=\frac{1}{N-1} \sum_{k=1}^{N-1} O_{q}^{i}(k+1)-O_{q}^{i}(k)^{(8)}
\end{aligned}
$$

With:

$i$ : is the index of the window.

$k:$ is the index of the period in the window.

$N$ : is number of periods in the window $i$.

$F_{0}^{i}(k)$ : is the instantaneous fundamental frequency of the kth period in the ith window.

$O_{q_{0}}^{i}(k)$ period in the ith window.

Multi-scale product is used for edge detection of pathological signal [33-36].

\section{SVM classifier}

Support vector machines have been used for the automatic classification of normal/pathological voices [37]. In the linearly separable case, the SVM optimization algorithm maximizes the margin between the two classes as shown in fig 3. In non-linearly separable cases, a mapping of the input data to some higher-dimensional space, where the data are linearly separable, is carried out by means of a Kernel function $\mathrm{K}$ that has to satisfy some properties called the Mercer conditions. The margin maximization algorithm leads to the following classifier, with $\mathrm{x}$ a data point to be assigned to one of two classes, according to the sign of the function in equation (9).

$f(x)=\sum_{i=1}^{N} \alpha_{i} y_{i} K\left(x, x_{i}\right)+b$

Where $y_{i} \in\{-1,1\}$ are the class labels, $b$ the bias term and

$\sum_{i=1}^{N} \alpha_{i} y_{i}=0, \alpha_{i}>0$

In this paper, a Gaussian kernel has been used.

$$
k(x, z)=\exp \left(-\frac{\|x-z\|^{2}}{2 \sigma^{2}}\right)
$$

Prior to training, inverse kernel width $\gamma$ and a penalty parameter $\mathrm{C}$ that is part of the cost function must be fixed. A larger $\mathrm{C}$ value corresponds to assigning a higher penalty to classification errors. A grid-search within intervals defined by the user is carried out to identify $(\mathrm{C}, \gamma)$ pairs that enable the classifier to predict unknown data as accurately as possible $\mathrm{C}=\left[10^{0}, 10^{3}\right]$ and $\gamma=\left[10^{-4}, 10^{-2}\right]$. The SVM toolbox Rouen software has been used for SVM training and classification [39].

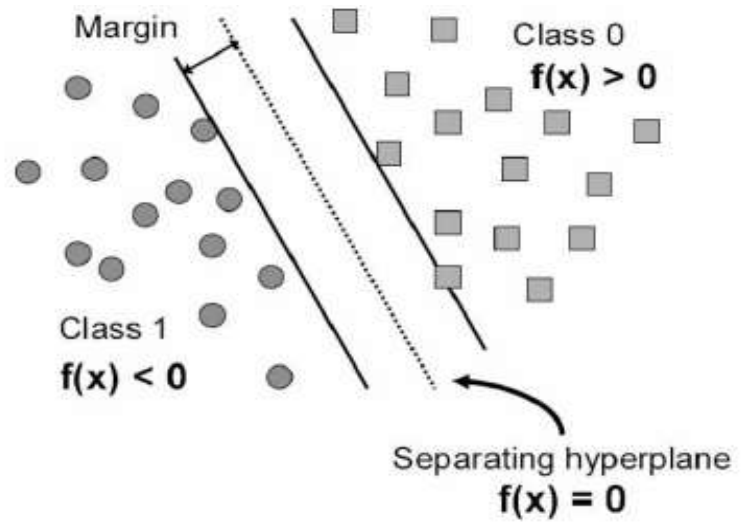

Figure 3. SVM classification: hyperplane maximizing the margin between two classes.

\section{A. Multi-category SVM classifier}

The one-against-all method has been used for multicategory classification in the framework of which one classifier is constructed for every pair of different classes [38, 39]. The total number of binary classifications is $K$ with $K$ the number of categories. The final decision is made using a majority rule. For each binary classification, the vote of the category in which the unknown sample has been classified is 
Proc. of the Second Intl. Conf. on Advances in Information Processing and Communication Technology - IPCT 2015 Copyright (C) Institute of Research Engineers and Doctors, USA .All rights reserved.

ISBN: 978-1-63248-044-6 doi: 10.15224/ 978-1-63248-044-6-120

incremented by one. The sample is assigned to the class with the largest vote [40].

\section{Ix. Simulations and Results}

The results of our work will be presented for MEII database. We operate two types of classification: a binary classification for all voices, a binary classification for female voices to provide a preliminary diagnosis concerning normal /pathologic voice and a classification into three classes normal/edema/nodule for female voices.

\section{A. Normal / pathologic voice classification for all speaker}

The normal / pathologic classification is also performed on the entire MEII database men and women using various combinations of parameters.

TABLE2. Confusing matrix of the normal / pathologic classification using all voices

\begin{tabular}{|c|c|c|c|}
\hline & & Normal & Pathologic \\
\hline \multirow[t]{2}{*}{ MFCC } & Normal & 99.65 & 0.35 \\
\hline & Pathologic & 11.26 & 88.74 \\
\hline \multirow[t]{2}{*}{$\mathrm{MFCC}+\Delta$} & Normal & 99.82 & 0.67 \\
\hline & Pathologic & 11.40 & 88.60 \\
\hline \multirow[t]{2}{*}{$\mathrm{MFCC}+\Delta \Delta$} & Normal & 99.65 & 0.35 \\
\hline & Pathologic & 11.26 & 88.74 \\
\hline \multirow[t]{2}{*}{$\mathrm{MFCC}+\Delta+\Delta \Delta$} & Normal & 99.82 & 0.18 \\
\hline & Pathologic & 11.26 & 88.74 \\
\hline \multirow[t]{2}{*}{$\begin{array}{l}\mathrm{MFCC}+\mathrm{E}+ \\
\Delta+\Delta \Delta\end{array}$} & Normal & 99.34 & 0.66 \\
\hline & Pathologic & 11.40 & 88.60 \\
\hline \multirow[t]{2}{*}{$\mathrm{MFCC}+\mathrm{F} 0$} & Normal & 96.16 & 3.84 \\
\hline & Pathologic & 16.69 & 83.31 \\
\hline \multirow[t]{2}{*}{$\mathrm{MFCC}+\mathrm{Oq}$} & Normal & 99.82 & 0.18 \\
\hline & Pathologic & 11.26 & 88.74 \\
\hline \multirow[t]{2}{*}{$\mathrm{MFCC}+\mathrm{F} 0+\mathrm{Oq}$} & Normal & 96.16 & 3.84 \\
\hline & Pathologic & 16.69 & 83.31 \\
\hline \multirow[t]{2}{*}{ All parameters } & Normal & 94.21 & 5.79 \\
\hline & Pathologic & 16.69 & 83.31 \\
\hline
\end{tabular}

As reported in table 2, we can see that all parameter combinations provide good results. However, the best rates are given with the MFCC coefficients only or associated with their first and second derivatives or with the open quotient Oq. The fundamental frequency drops the recognition of pathological voices from $88 \%$ to $83 \%$. With all the parameters, the results are lightly worse than with F0.

\section{B. Normal / pathologic voice classification for all female speakers}

The normal / pathologic classification is performed on the women MEII database using various combinations of parameters.

TABLE3. Confusing matrix of the normal / pathologic classification using all female voices

\begin{tabular}{|c|c|c|c|}
\hline & & Normal & Pathologique \\
\hline \multirow[t]{2}{*}{ MFCC } & Normal & 98 & 2 \\
\hline & Pathologic & 0,35 & 99,65 \\
\hline \multirow[t]{2}{*}{$\mathrm{MFCC}+\Delta$} & Normal & 98 & 2 \\
\hline & Pathologic & 0,35 & 99,65 \\
\hline \multirow[t]{2}{*}{$\mathrm{MFCC}+\Delta \Delta$} & Normal & 98 & 2 \\
\hline & Pathologic & 0,35 & 99,65 \\
\hline \multirow[t]{2}{*}{$\mathrm{MFCC}+\Delta+\Delta \Delta$} & Normal & 98 & 2 \\
\hline & Pathologic & 0,35 & 99,65 \\
\hline \multirow[t]{2}{*}{$\begin{array}{l}\text { MFCC+E+ } \\
\Delta+\Delta \Delta\end{array}$} & Normal & 97,83 & 2.17 \\
\hline & Pathologic & 0 & 100 \\
\hline \multirow[t]{2}{*}{$\overline{\mathrm{MFCC}+\mathrm{F}_{0}}$} & Normal & 92,8 & $\overline{7,2}$ \\
\hline & Pathologic & 0 & 100 \\
\hline \multirow[t]{2}{*}{$\mathrm{MFCC}+\mathrm{O}_{\mathrm{q}}$} & Normal & 98,26 & 1.74 \\
\hline & Pathologic & 0.35 & 99.65 \\
\hline \multirow[t]{2}{*}{$\mathrm{MFCC}+\mathrm{F}_{0}+\mathrm{O}_{\mathrm{q}}$} & Normal & 89.15 & 10.85 \\
\hline & Pathologic & 0 & 100 \\
\hline \multirow[t]{2}{*}{ All parameters } & Normal & 88.54 & 11.46 \\
\hline & Pathologic & 0 & 100 \\
\hline
\end{tabular}

As reported in table 3, we can see that all the best rates are given with the MFCC coefficients only or associated with their first and second derivatives or associated with the open quotient Oq. The fundamental frequency drops the recognition of pathological voices. With all the parameters, the results are lightly worse than with F0. 
Proc. of the Second Intl. Conf. on Advances in Information Processing and Communication Technology - IPCT 2015 Copyright $($ Institute of Research Engineers and Doctors, USA .All rights reserved.

ISBN: 978-1-63248-044-6 doi: 10.15224/ 978-1-63248-044-6-120

\section{Triple classification edema/nodule/normal for all women of the MEII database}

In this classification we use 14 edema, 14 nodule, and 14 normal voices. The classification rates are reported on table 4 .

TABLE4. Confusing matrix for the classification in the MEII database of edema, nodule, and normal female voices using a 3-class SVM

\begin{tabular}{|c|c|c|c|c|}
\hline & & Edema & Nodule & Normal \\
\hline $\mathrm{MFCC}$ & $\begin{array}{l}\text { Edema } \\
\text { Nodule } \\
\text { Normal }\end{array}$ & $\begin{array}{l}73.38 \\
12.57 \\
0\end{array}$ & $\begin{array}{l}26.62 \\
74.85 \\
0\end{array}$ & $\begin{array}{l}0 \\
12.58 \\
100\end{array}$ \\
\hline $\mathrm{MFCC}+\Delta$ & $\begin{array}{l}\text { Edema } \\
\text { Nodule } \\
\text { Normal }\end{array}$ & $\begin{array}{l}73.38 \\
12.57 \\
0 \\
\end{array}$ & $\begin{array}{l}26.62 \\
74.85 \\
0 \\
\end{array}$ & $\begin{array}{l}0 \\
12.58 \\
100 \\
\end{array}$ \\
\hline $\mathrm{MFCC}+\Delta \Delta$ & $\begin{array}{l}\text { Edema } \\
\text { Nodule } \\
\text { Normal }\end{array}$ & $\begin{array}{l}73.38 \\
12.57 \\
0 \\
\end{array}$ & $\begin{array}{l}26.62 \\
74.85 \\
0 \\
\end{array}$ & $\begin{array}{l}0 \\
12.58 \\
100 \\
\end{array}$ \\
\hline $\begin{array}{l}\text { MFCC+ } \\
\Delta+\Delta \Delta\end{array}$ & $\begin{array}{l}\text { Edema } \\
\text { Nodule } \\
\text { Normal }\end{array}$ & $\begin{array}{l}73.38 \\
12.57 \\
0\end{array}$ & $\begin{array}{l}26.62 \\
74.85 \\
0\end{array}$ & $\begin{array}{l}0 \\
12.58 \\
100\end{array}$ \\
\hline $\begin{array}{l}\mathrm{MFCC}+\mathrm{E}+ \\
\Delta+\Delta \Delta\end{array}$ & $\begin{array}{l}\text { Edema } \\
\text { Nodule } \\
\text { Normal }\end{array}$ & $\begin{array}{l}78.42 \\
12.57 \\
0\end{array}$ & $\begin{array}{l}21.58 \\
74.25 \\
0 \\
\end{array}$ & $\begin{array}{l}0 \\
13.18 \\
100\end{array}$ \\
\hline $\mathrm{MFCC}+\mathrm{F} 0$ & $\begin{array}{l}\text { Edema } \\
\text { Nodule } \\
\text { Normal }\end{array}$ & $\begin{array}{l}64.74 \\
31.14 \\
0 \\
\end{array}$ & $\begin{array}{l}31.65 \\
40.71 \\
0 \\
\end{array}$ & $\begin{array}{l}3.60 \\
28.15 \\
100 \\
\end{array}$ \\
\hline $\mathrm{MFCC}+\mathrm{Oq}$ & $\begin{array}{l}\text { Edema } \\
\text { Nodule } \\
\text { Normal }\end{array}$ & $\begin{array}{l}40.95 \\
52.15 \\
1.94 \\
\end{array}$ & $\begin{array}{l}55.12 \\
24.64 \\
0 \\
\end{array}$ & $\begin{array}{l}3.93 \\
23.21 \\
98.06 \\
\end{array}$ \\
\hline $\begin{array}{l}\mathrm{MFCC}+\mathrm{F} 0+ \\
\mathrm{Oq}\end{array}$ & $\begin{array}{l}\text { Edema } \\
\text { Nodule } \\
\text { Normal }\end{array}$ & $\begin{array}{l}67.62 \\
33.53 \\
0\end{array}$ & $\begin{array}{l}32.38 \\
61.08 \\
1.24 \\
\end{array}$ & $\begin{array}{l}0 \\
5.39 \\
98.76 \\
\end{array}$ \\
\hline $\begin{array}{l}\text { All } \\
\text { parameters }\end{array}$ & $\begin{array}{l}\text { Edema } \\
\text { Nodule } \\
\text { Normal }\end{array}$ & $\begin{array}{l}100 \\
20.96 \\
13.65\end{array}$ & $\begin{array}{l}0 \\
79.04 \\
0\end{array}$ & $\begin{array}{l}0 \\
0 \\
86.35\end{array}$ \\
\hline
\end{tabular}

We note that the best recognition is obtained with all parameters. The MFCC coefficients used alone recognize $100 \%$ of the normal speakers, the edema with $73.39 \%$ and the nodule with $74.85 \%$. The open quotient Oq influence on edema and nodule classes by lowering the recognition of edema to $64.74 \%$ and the nodule to $40.71 \%$, against the recognition of normal voices remains effective.

\section{$x$. Performance of the classification system}

In order to quantify the classifier performance, we consider three measures: sensitivity, specificity, and the overall accuracy. These measures are calculated from:

- the true positive (TP: the classifier classified as pathology when pathological samples are present),

- $\quad$ true negative (TN: the classifier classified as normal when normal samples are present),

- false positive (FP: the classifier classified as pathological when normal samples are present),

- $\quad$ false negative (FN: the classifier classified as normal when pathological samples are present).

These measures are calculated using the following equations

$$
\begin{aligned}
& \text { Sensitivity }(\mathrm{SE})=\frac{T P}{T P+F N} \\
& \operatorname{Specificity}(\mathrm{SP})=\frac{T N}{T N+F P}
\end{aligned}
$$

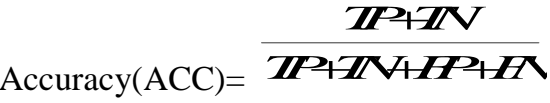

These parameters are extracted from the confusion matrix as follows in table 5 .

TABLE5. The structure of a confusion matrix

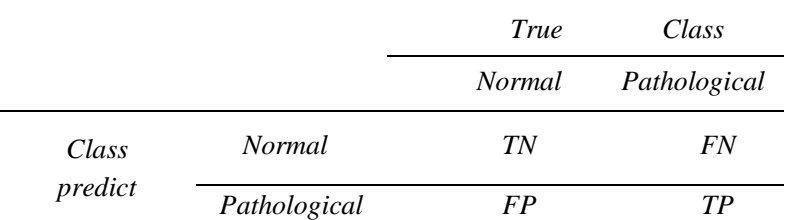

\section{A. Performance of normal/ pathologic classification for all speakers}

Table 6 illustrates the sensitivity, specificity and accuracy values for selected parameters for the classification of normal and pathologic voices in the MEII database.

TABLE6. Performance of normal / pathological classification of all speakers of MEII database

\begin{tabular}{lccc}
\hline & $\begin{array}{c}\text { Sensibility } \\
\%\end{array}$ & $\begin{array}{c}\text { Specificity } \\
\%\end{array}$ & $\begin{array}{c}\text { Accuracy } \\
\%\end{array}$ \\
\hline $\boldsymbol{M F C C}$ & $\mathbf{9 9 . 6}$ & $\mathbf{8 9 . 8 4}$ & $\mathbf{9 4 . 1 9}$ \\
MFCC $+\boldsymbol{\Delta}+\boldsymbol{\Delta A}$ & $\mathbf{9 9 . 8}$ & $\mathbf{8 9 . 8 6}$ & $\mathbf{9 4 . 2 8}$ \\
MFCC $+F_{0}$ & 95.59 & 85.21 & 89.73 \\
$\boldsymbol{M F C C}+\boldsymbol{O}_{q}$ & $\mathbf{9 9 . 8}$ & $\mathbf{8 9 . 8 6}$ & $\mathbf{9 4 . 2 8}$ \\
All parameters & 95.51 & 84.95 & 88.76 \\
\hline
\end{tabular}


The classification using the MFCC coefficients alone or with their first and second derivatives and the combination $\mathrm{MFCC}+\mathrm{Oq}$ give the highest rates.

The classifications using the MFCC coefficients + F0 or all parameters give the worst results.

\section{B. Performance of normal/ pathologic classification for female speakers}

Table 7 illustrates the sensitivity, specificity and accuracy values for selected parameters for the classification of normal and pathologic of female voices in the MEII database.

TABLE7. Performance of normal / pathological classification for female speakers of MEII database

\begin{tabular}{llll}
\hline & Sensibility $\%$ & Specificity $\%$ & Accuracy $\%$ \\
\hline MFCC & $\mathbf{9 8 , 0 3}$ & $\mathbf{9 9 , 6 4}$ & $\mathbf{9 8 , 3 2}$ \\
MFCC $+\boldsymbol{\Delta}+\boldsymbol{\Delta} \boldsymbol{\Delta}$ & $\mathbf{9 7 , 8 7}$ & $\mathbf{1 0 0}$ & $\mathbf{9 8 , 9 2}$ \\
MFCC $+\mathrm{F}_{0}$ & 93,28 & 100 & 96,0 \\
MFCC+ $\mathbf{O}_{\mathbf{q}}$ & $\mathbf{9 8 , 2 8}$ & $\mathbf{9 9 , 6 4}$ & $\mathbf{9 8 , 9 5}$ \\
All parameters & 89,72 & 100 & 94,27 \\
\hline
\end{tabular}

The classification using the MFCC coefficients alone or with their first and second derivatives and the combination MFCC + Oq give the highest rates.

The classifications using the MFCC coefficients $+\mathrm{F}_{0}$ or all parameters give the worst results in terms of sensibility.

\section{Performance of classification edema/nodule/normal female voices}

The triple classification edema / nodule / normal for women voices by a 3-class SVM using MEII database is presented in table 8 .

The combination of all parameters has the highest accuracy rate. The combinations MFCC + F0 and MFCC + Oq have the less accuracy.

TABLE8. Accuracy of the classification (edema, nodule, and normal) for female speakers

\begin{tabular}{ll}
\hline & Accuracy \% \\
\hline MFCC & 82.33 \\
MFCC $+\Delta+\Delta \Delta$ & 82.33 \\
MFCC + F0 & 68 \\
MFCC + Oq & 54 \\
All parameters & 88.33 \\
\hline
\end{tabular}

\section{Comparison of the Performance of normal / pathologic classification system with other works}

We compare the best performance of MFCC + Oq to those proposed by Hariharan, Polatb, Sindhuc, Yaacoba in [41], Arias-Londono, Godino-Llorente, Markaki, Stylianou in [42] and Alpan, Schoentgen, Maryn, Grenez in [43] as shown in table 9 .

TABLE9. MFCC+Oq performances compared with other works for normal / pathological classification

\begin{tabular}{|c|c|c|c|c|c|c|}
\hline \multirow{2}{*}{$\begin{array}{l}\text { Author } \\
\text { ACC \% }\end{array}$} & \multicolumn{2}{|c|}{ Method } & Parameters & \multirow{2}{*}{\begin{tabular}{|l} 
Years \\
95.20
\end{tabular}} & \multirow{2}{*}{$\begin{array}{l}S E \% \\
91.04\end{array}$} & \multirow{2}{*}{\begin{tabular}{|c|}
$S P \%$ \\
94.22 \\
\end{tabular}} \\
\hline & $G M M$ & $12 M F C C$ & 2010 & & & \\
\hline \multicolumn{7}{|l|}{ [42] } \\
\hline & $S V M$ & $M S$ & 2010 & 97.38 & 79.72 & 93.22 \\
\hline & $S V M$ & $S D R$ & 2010 & 98.2 & 97.9 & 98.1 \\
\hline [43] & $S V M$ & $\begin{array}{l}M F C C+E \\
+\Delta+\Delta \Delta\end{array}$ & 2010 & 98.7 & 94 & 97.6 \\
\hline \multirow{3}{*}{ [41] } & $S V M$ & $P C A$ & 2013 & 92.01 & 90.29 & 91.12 \\
\hline & $S V M$ & $L D A$ & 2013 & 90.22 & 89.05 & 89.61 \\
\hline & $S V M$ & $P C A / F C M$ & 2013 & 100 & 99.95 & 99.98 \\
\hline $\begin{array}{c}\text { Our } \\
\text { App- } \\
\text { roach }\end{array}$ & $S V M$ & $M F C C+O_{q}$ & 2013 & 99.8 & 89.86 & 94.28 \\
\hline
\end{tabular}

In terms of sensitivity, our approach and the one using SVM with the PCA / FCM parameters are the best.

In terms of specificity, our approach is better than the SVM +MS and SVM +LDA systems, but it lags behind the rest.

In terms of accuracy, our approach is more efficient than GMM+12 MFCC, SVM + MS and SVM+ LDA only.

\section{Conclusion}

This paper presents the evaluation of the behavior of our proposed classification system applied on pathological voices and depending on the parameterisation. Our contribution 
concerns the addition of a new parameter to the classical parameters formed by MFCC coefficients, the energy, their first and second derivatives and F0 and the study of the effect of this parameter in the classification performances. Besides, we consider a binary classification between normal and pathological voices for all speakers and a triple classification between normal, edema and nodule voices for female speakers only.

The Open quotient and the fundamental frequency are computed from the glottal opening instant GOI and the glottal closing instant GCI localised by the multi-scale product (MP). The classification is performed by an SVM multiclass system according to one against all approach using the gaussian kernel. The proposed approach is tested on MEII database of pathological voices.

For all these classifications, we vary the set of parameters to investigate the relative effect of the fundamental frequency and the open quotient on the classification rates.

In the MEII database, the classification by SVM two classes normal / pathological for all speakers, the MFCC coefficients significantly improve the classification rate. When adding the Oq or $\Delta+\Delta \Delta$ to MFCC coefficients, the recognition rate remains high. As against, the parameter F0 drops the recognition of patients from $88 \%$ to $83 \%$.

For the 3-class SVM classification into edema, nodule and normal for women, the best recognition is obtained with all parameters. The MFCC coefficients allow to recognize 100\% of normal speakers, the edema disease to $73.39 \%$ and nodule disease to $74.85 \%$. The open quotient $\mathrm{Oq}$ deteriorates the recognition of the 2 diseases.

Future works concern the classification into between pathologies in the MEII database and testing other parameters extracted from the speech multi-scale product.

\section{References}

[1] J.I. Godino-Llorente, P. Gomez-Vilda, and T. Lee, "Analysis and Signal Processing of Oesophageal and Pathological Voices", EURASIP Journal on Advances in Signal Processing, Special Issue on Analysis and Signal Processing of Oesophageal and Pathological Voices, 2009.

[2] J.I. Godino-Llorente, P. Gomez-Vilda, N. Saenz-Lechon, M. BlancoVelasco, F. Cruz Roldan, and M.A. Ferrer, "Discriminative methods for the detection of voice disorders", In: NOLISP 2005 International Conference on Non-Linear Speech Processing, April 2005; Barcelona, Spain.

[3] J.I. Godino-Llorente, R. Fraile, N. Saenz-Lechon, V. Osma-Ruiz, and P. Gomez-Vilda, "Automatic Detection of Voice Impairments from TextDependent Running Speech using a Discriminative Approach", In: MAVEBA 2007, pp. 25-28.

[4] M. Hirano, "Psycho-Acoustic Evaluation of Voice: GRBAS Scale for Evaluation the Horse Voice", Springer 1981; Berlin, Germany.

[5] K. Marasek, "An Attempt to classify lx signals", In: EuroSpeech 1995 the 4th European Conference on Speech Communication and Technology; September 1995; Madrid, Spain.

[6] D. Deliyski, "High-speed videoendoscopy: recent progress and clinical prospects", In: AQL 2006 the 7th International Conference on Advances in Quantitative Laryngology Voice and Speech Rearch , 2006; University of Groningen, pp.1-12.

[7] J. Demeyer, and B. Gosslin, "Glottis segmentation with a high speed glottography: a new approach", In Proceedings of Liege Image Days, March 2008; Liege, Belgium .

[8] J.I. Godino-Llorente, P. Gomez Vilda, N. Saenz-Lechon1, M. BlancoVelasco, F. Cruz-Roldan, and M. Angel Ferrer-Ballester, "Support Vector
Machines Applied to the Detection of Voice Disorders", Springer-Verlag, Berlin Heidelberg, 2005. pp.219-230.

[9] J. Iagnacio Godino-Llorente, Member, IEEE, P. Gomez Vilda , Member, IEEE, M. Blanco-Velasco, Member, IEEE, "Dimensionality Reduction of a Pathological Voice Quality Assesment System Based on Gaussian Mixture Models and Short-Term Cepstral Parameters", In: IEEE 2006 Transactions on Biomedical Engeneering, October 2006; 5.

[10] A.A. Dibazar, T.W. Berger, and S.S. Narayanan, "Pathological Voice Assesment", In: IEEE 2006 EMBS 2006; New York.

[11] J. Benesty, M.M. Sondhi, and Y. Huang, "Springer Handbook of Speech Processing", Springer, Berlin, Germany 2008.

[12] F. Servin, B. Bozkurt, and T. Dutoit, "Hnr extraction in voiced speech oriented towards voice quality analysis", In: EUSIPCO 2005 13th European Signal Processing Conference; September 2005; Antalya, Turkey.

[13] G. De Krom, "Spectral correlates of breathiness and roughness for different types of vowel fragments", In:ICSLP 1994 the 3rd International Conference on Spoken Language Processing; September 1994; Yokohama, Japan.

[14]C. D'Alessandro,F. Yegnanarayana, and A. Darsinos, «Decompositions », In: ICASSP 1993 the IEEE International Conference on Acoustics, Speech, and Signal; May 1993; Detroit, Mich, USA: pp.760-763.

[15] P. Boersma, "Accurate short-term analysis of the fundamental frequency and the harmonics-to-noise ratio of a sampled sound", In: IFA1993 of the Institute of Phonetic Sciences; 1993; Amsterdam, The Netherlands.

[16] K. Shama, A. Krishna, and N.U. Cholayya, "Study of harmonics-to-noise ratio and critical-band energy spectrum of speech as acoustic indicators of laryngeal and voice pathology", EURASIP Journal on Advances in Signal Processing 2007; Article ID 85286, 9.

[17] M. Wester, "Automatic classification of voice quality: comparing regression models and hidden markov models", In: VOICEDATA 1998 Symposium on Databases in Voice Quality Research and Education;1998.

[18]R.B. Reilly, R. Moran, and P. Lacy, "Voice pathology assessment based on a dialogue system and speech analysis", In: AAAI 2004 Symposium on Dialogue Systems for Health Communication; 2004; pp. 104-109.

[19] R.J. Moran, R.B. Reilly, P. de Chazal, and P.D. Lacy, "Telephony-based voice pathology assessment using automated speech analysis", IEEE Transactions on Biomedical Engineering; 2006; 3:468-477.

[20] Corp KE. Multi-dimensional voice program (mdvp) [computer program]. Tech Rep, Kay Elemetrics Corp, 2008.

[21] Corp K. E. Disordered voice database model (version 1.03). Tech Rep, Massachussets Voice Eye and Ear Infirmary Voice and Speech Lab, 1994.

[22] A. Dibazar, S. Narayanan, "A system for automaticdetection of pathological speech", In: 36th Asilomar 2002 Conference on Signals, Systems and Computers; November 2002; Pacific Grove, Calif, USA.

[23] J.I. Godino-Llorente, S. Aguilera-Navarro, C. Hernandez- Espinosa, M. Fernandez-Redondo, and P. Gomez-Vilda, "On the selection of meaningful speech parameters used by a pathologic/non pathologic voice register classifier", In: EUROSPEECH 1999 the 6th European Conference on Speech Communication and Technology; September 1999; Budapest, Hungary.

[24]C. Fredouille, G. Pouchoulin, J.F. Bonastre, M. Azzarello, A. Giovanni, and A. Ghio, "Application of automatic speaker recognition techniques to pathological voice assessment (dysphonia)", In: EuroSpeech 2005, the 9th European Conference on Speech Communication and Technology; September 2005; Lisbon, Portugal, pp. 149-152.

[25]G. Pouchoulin, C. Fredouille, J. Bonastre , A. Ghio, M. Azzarello, and A. Giovanni, «Modélisation statistique et informations pertinentes pour la caractérisation des voix pathologiques (dysphonies) », In : JEP 2006 (Journée d'Etudes sur la Parole); 2006.

[26]C. Bishop, "Pattern Recognition and Machine Learning", Springer, New York, NY, USA, 2006.

[27]P. Kukharchik, I. Kheidorov, E. Bovbel, and D. Ladeev, Image and signal processing, In: Speech Signal Processing Based on Wavelets and SVM for Vocal Tract Pathology Detection, Lecture Notes in Computer Science Springer; Berlin, Germany, pp. 192-199, 2008.

[28]J.I. Godino-Llorente, S. Aguilera-Navarro, C. Hernandez-Espinosa, M. Fernandez-Redondo, and P. Gomez-Vilda, "On the selection of meaningful speech parameters used by a pathologic/non pathologic voice register classifier". In: EUROSPEECH 1999 the 6th European Conference on Speech Communication and Technology; September 1999; Budapest, Hungary. 
[29]J.B. Alonso, J. de Leon, I. Alonso, and M.A. Ferrer, "Automatic detection of pathologies in the voice by HOS based parameters". EURASIP Journal on Advances in Signal Processing 2001; 4: pp. 275-284.

[30]Kay Elemetrics Inc. Voice disorders database, version 1.03[CDROM][Online].Available:http://www.kaypentax.com/Product\%20Inf o/CSL\%20Options/4337/4337.htm.

[31] MAPACI P. Voice Disorder Database [Online]. Available: http:// www. Mapaci.com/index-ingles.php

[32] http://www.ee.ic.ac.uk/hp/staff/dmb/voicebox/voicebox.html

[33]A. Belhaj, A. Bouzid, N. Ellouze, and A. Nait-ali, « Paramétrisation des voix pathologiques à partir du MPM et leurs classifications », Quatrièmes journées de phonétique clinique ;2011; Strasbourg, France.

[34]A. Belhaj, A. Bouzid, N. Ellouze, and A. Nait-ali, "Disordered voice parametrisation using the multi-scale product", In : SETIT 2012Sciences of Electronics, Technologies of Information and Telecommunications; Mars 2012 ;Sousse, Tunis.

[35]A. Belhaj, A. Bouzid, N. Ellouze, "Statistical voicing parameter analysis of pathological signals using the Multi-scale Product and SVM classification", In: ATSIP 2014 International Conference on Advanced Technologies for Signals \& Image processing; Mars 2014; Sousse,Tunis.

[36]S. Chekili, A. Belhaj, A. Bouzid, N. Ellouze, "Recognition of pathological voices", In: IEEE 2014 International Multi-Conference on Systems, Signals \& Devices, Conference on Communication \& Signal Processing; Février 2014; Barcelona, Espagne.

[37]V. Vapnik, "An overview of statistical learning theory", In: IEEE 1999 Transactions on Neural Networks; September 1999; IEEE. pp. 988-1000.

[38] http://asi.insarouen.fr/enseignants/ arakoto/toolbox/index.html.

[39] C.W. Hsu, and C.J. Lin, "A comparison of methods for multi-class support vector machine", In: IEEE 2002Transactions on Neural Networks; 2002; IEEE. pp.415-425.

[40]J. Friedman, "Another Approach to Polychotomous Classification", Technical report, Department of Statistics, Stanford University, 1996.

[41] M. Hariharan, K. Polatb, R. Sindhuc and S. Yaacoba, "A hybrid expert system approach for telemonitoring of vocal fold pathology", Applied Soft Computing 13, pp. 4148-4161, 2013.

[42] J. D. Arias-Londono, J. I. Godino-Llorente, M. Markaki, Y. Stylianou and Y. Stylianou, "On combining information from modulation spectra and mel-frequency cepstral coefficients for automatic detection of pathological voices", Logopedics Phoniatics Vocoly, 2010.

[43] A. Alpan, J. Schoentgen, Y. Maryn and F. Grenez, "Automatic Perceptual Categorization of Disordered Connected Speech", In Proceedings of Interspeech, pp. 2574-257, 2010. 